\title{
Composición de la flora de praderas andinas en la Reserva Nacional Alto Bío-Bío (Lonquimay-Chile) y su relación con el régimen de pastoreo
}

\section{Floristic composition of Andean grassland in Alto Bío-Bío National Reserve (Lonquimay- Chile) and its relationship with the grazing regimen}

\author{
Andrés Fuentes-Ramírez ${ }^{1-2}$, Aníbal Pauchard ${ }^{1-2}$ \& Enrique Hauenstein ${ }^{3}$ \\ ${ }^{1}$ Laboratorio de Invasiones Biológicas (LIB), Facultad de Ciencias Forestales, Universidad de Concepción. Casilla 160-C, \\ Concepción, Chile. \\ ${ }^{2}$ Instituto de Ecología y Biodiversidad (IEB). Facultad de Ciencias, Universidad de Chile, Casilla 653, Santiago, Chile. \\ ${ }^{3}$ Escuela de Ciencias Ambientales, Facultad de Recursos Naturales, Universidad Católica de Temuco. Casilla 15-D, Temuco- \\ Chile. \\ andresfuen@yahoo.es
}

\section{RESUMEN}

\begin{abstract}
Las praderas andinas de la Región de La Araucanía son usadas estacionalmente por comunidades Pehuenches como forraje para sus animales. El pastoreo es una de las principales perturbaciones que afectan a las praderas naturales, pudiendo generar cambios a nivel comunitario en la composición y en la riqueza de especies. Con el objetivo de determinar la composición de la flora de las praderas andinas de la Reserva Nacional Alto Bío-Bío $\left(37^{\circ} \mathrm{S}\right.$ y $\left.70^{\circ} \mathrm{O}\right)$ y su relación con la carga animal que soporta cada una, se estudiaron cinco sitios al interior de la reserva: Cuchares Grande, Cajón Chileno, Tralilhue, Cuchares Chico y Mancha de Pinos. En cada sitio se realizaron 20 parcelas al azar, registrándose todas las especies presentes y su cobertura relativa. En total se registraron 89 especies de plantas vasculares. La riqueza taxonómica está representada por dos especies de Pteridophyta y 87 de Magnoliophyta, de las cuales 25 son Liliopsida y 62 Magnoliopsida. Las 89 especies se distribuyen en 72 géneros y 37 familias, siendo las más numerosas Asteraceae (13), Poaceae (11), Cyperaceae (6) y Rosaceae (6). De las especies determinadas, un $88 \%$ son nativas y 12\%, introducidas. Las formas de vida están representadas principalmente por hemicriptófitos con un 53\%, criptófitos (17\%) y caméfitos (15\%). Las praderas que tienen mayor carga ganadera son las que presentan mayor proporción de especies introducidas y hemicriptófitas, existiendo mayor similitud florística entre ellas, mientras que las que tienen menor carga ganadera presentan mayor proporción de especies nativas. Estos resultados sugieren que el uso ganadero podría tener efectos importantes en el cambio de la composición de la flora de las praderas andinas. A pesar de la baja proporción de especies introducidas en total, el alto porcentaje de hemicriptófitos indicaría un grado medio de perturbación.
\end{abstract}

Palabras clave: Veranadas, Alto Bío-Bío, riqueza de especies, perturbación, sobrepastoreo.

\begin{abstract}
Andean grasslands of the La Araucanía Region are used every year by Pehuenches communities as forage resources for their livestock. Grazing is one of the most important disturbances which affect natural meadows and can produce changes at community level in the species richness and plant composition. With the aim of determining the floristic composition of the Andean grasslands in the Alto Bío-Bío National Reserve $\left(37^{\circ} \mathrm{S}\right.$ y $\left.70^{\circ} \mathrm{W}\right)$ and its relationship with the cattle load, we studied five sites within the reserve: Cuchares Grande, Cajón Chileno, Tralilhue, Cuchares Chico and Mancha de Pinos. In each site, we sampled 20 random plots recording all the species and their relative cover. We recorded a total of 89 species of vascular plants. Taxonomic richness is represented by two Pteridophyta and 87 Magnoliophyta species. In this last group, 25 species are Liliopsida and 62 are Magnoliopsida. All flora is distributed in 37 families and 72 genera. Greatest families, in species number, are Asteraceae (13), Poaceae (11), Cyperaceae (6) and Rosaceae (6). About the origin, 88 species are native and only 11 are introduced, representing $88 \%$ and $12 \%$, respectively. Life forms are represented mostly by hemicryptophytes with $53 \%$, then cryptophytes arround $17 \%$ and finally chamaephytes (15\%). Grasslands wich have greater livestock density, have a higher proportion of introduced species and hemicryptophytes and a greater floristic similarity between them, while those with lower livestock densities have a higher native species proportion. These results suggest that cattle use could have a significant impact in the floristic composition of Andean grasslands. Even though, the low proportion of introduced species found, the high percentage of hemicryptophytes indicates an intermediate level of disturbance.
\end{abstract}

KeYwords: Summer pastures, Alto Bío-Bío, species richness, disturbance, overgrazing. 


\section{INTRODUCCIÓN}

Las praderas constituyen un eje central en el desarrollo ganadero del país, incorporando praderas artificiales $\mathrm{y}$ naturales desde la cordillera andina hasta el secano costero, las cuales cubren una superficie aproximada de 13 millones de hectáreas (SAG 2004). En Chile, las praderas andinas se distribuyen a lo largo de toda la cordillera de los Andes (Ahumada et al. 1999). Se encuentran desde el extremo norte asociadas a bofedales, en el centro asociadas a un intenso uso ganadero, y en el extremo sur del país como extensos pastizales en las regiones de Aisén y Magallanes (Bustamante 2007). En la Región de La Araucanía, la cordillera de los Andes presenta un doble alineamiento montañoso: el cordón limítrofe al este, con forma de meseta y con alturas $<2.000$ msnm, y el cordón exterior u occidental, volcánico con cumbres cónicas $>2.000$ msnm. Entre ambos cordones se encuentra el amplio valle del Alto Bío-Bío y sus principales afluentes (IGM 1986). En estas cumbres se encuentran las praderas andinas (también llamadas veranadas), que corresponden a praderas naturales que poseen un uso ganadero estacional en los meses de verano, ya que en los meses de invierno permanecen cubiertas de nieve (Hauenstein et al. 2003).

Las comunidades vegetales de las zonas andinas de la Región de La Araucanía corresponden a bosques de Araucaria araucana (Molina) K.Koch como especie dominante y representativa del área, Nothofagus pumilio (Poepp. et Endl.) Krasser, Nothofagus antarctica (G.Forst.) Oerst., Nothofagus dombeyi (Mirb.) Oerst. y matorrales de Discaria chacaye (G.Don) Tortosa (Hauenstein et al. 2003). A su vez, las veranadas están formadas por gramíneas y hierbas que crecen en las vegas o mallines, que en su mayoría son pastoreadas por caprinos, equinos, ovinos y vacunos (Ahumada et al. 1999). Son ecosistemas frágiles que se encuentran en las cabeceras de las cuencas hidrográficas y cumplen una función importante en el ciclo hidrológico. Además, constituyen una fuente de biodiversidad de plantas y animales propios de ecosistemas andinos (Ventura 2001), cuyas especies presentan extraordinarias adaptaciones a las condiciones de temperaturas extremas (Larcher et al. 2010). Tienen importancia geopolítica, ecológica, turística, económica y cultural (Peralta 1980, López 1990, Mardones et al. 1993, Hoffmann et al. 1998, Molina \& Correa 1998, Tacón et al. 2002, CONAF 2000, 2004, Florez 2005). En La Araucanía andina estas veranadas se encuentran sobre la cota de los 900 msnm en las comunas de Curarrehue, Melipeuco y Lonquimay (Pérez 2001).

Las veranadas del sector cordillerano de La Araucanía han sido utilizadas ancestralmente por comunidades Pehuenche de la zona, existiendo indicios de ocupación desde hace más de 450 años (Mardones et al. 1993). Respecto de colonos chilenos, se tienen antecedentes de su llegada a la comuna de Lonquimay y otros sectores hace más de 100 años (Molina \& Correa 1998).
Desde el punto de vista ganadero, hasta mediados de los años 90 gran parte de estas zonas eran controladas rigurosamente por el Servicio Agrícola y Ganadero (SAG) para evitar el contagio con la fiebre aftosa proveniente de Argentina. En la actualidad las condiciones zoosanitarias han mejorado en ambos países, lo que ha traído consigo un aumento en la presión de uso de los recursos pratenses de las veranadas (Ahumada et al. 1999). De hecho, el número de vacunos ingresados a las veranadas de la Reserva Nacional Alto Bío-Bío prácticamente se duplicó en el período 20002008 (Fuentes-Ramírez com. pers.). El pastoreo es una de las principales perturbaciones que afectan a las praderas naturales, pudiendo generar cambios a nivel comunitario en la riqueza, composición de las especies nativas e introducidas y en la diversidad de las comunidades (Stohlgren et al. 1999a, Davis et al. 2000, Adler et al. 2005). Además, puede alterar el espectro de formas de vida de las plantas y su cobertura, incrementar la llegada de especies introducidas y favorecer con ello el proceso de invasión de especies alóctonas en la comunidad nativa (Pauchard et al. 2004, Pykälä 2004, Castro et al. 2010).

Los trabajos documentados sobre esta temática en Chile son escasos, destacando a López (1990), quien utilizando imágenes satelitales estructuró satelogramas que incluían clases temáticas específicas de distintas coberturas de vegetación. Más tarde, Ugarte \& Barrientos (1991) y Ugarte et al. (1993) estudiaron la vegetación de la cuenca del Alto Bío-Bío. Ahumada et al. (1999), a su vez, describieron las pautas de condición y capacidad sustentadora de las veranadas de la IX Región y finalmente Hauenstein et al. (2003) establecieron la composición de la flora de siete veranadas del sector norte de la comuna de Lonquimay, no incluyendo por tanto las praderas del presente estudio, que se ubican en el sector sur de dicha comuna (Fig. 1).

En este trabajo se plantea la hipótesis que, de acuerdo con los antecedentes conocidos (Ahumada et al. 1999, Hauenstein et al. 2003), las praderas andinas con uso ganadero más intenso tendrían una mayor proporción de especies de plantas vasculares introducidas y de formas de vida adaptadas al pastoreo, comparadas con aquellas praderas de uso menos intenso. Con el objeto de contribuir al conocimiento y manejo de este importante recurso, el presente trabajo describe la composición de la flora vascular de las veranadas de la Reserva Nacional Alto Bío-Bío y su relación con la carga animal que soporta cada una de ellas.

\section{MATERIALES Y MÉTODOS}

ÁreA DE ESTUDiO

El área de estudio está ubicada en la Reserva Nacional Alto Bío-Bío ( $37^{\circ} \mathrm{S} ; 70^{\circ} \mathrm{W}$ ), provincia de Malleco, comuna de Lonquimay. La Reserva cuenta con una superficie de 35.525 ha y se encuentra a $186 \mathrm{~km}$ de la ciudad de Temuco por el 
camino a Lautaro (Fig. 1). Según la clasificación de Koeppen (1948), en la zona se pueden encontrar tres tipos de clima: el EFH (de hielo por efecto de la altura) correspondiente a Lonquimay, al poniente un clima Cfsb1 (templado cálido con menos de 4 meses secos), y un sector oriental en el límite con Argentina del tipo BSK (de estepa fría). La temperatura media anual es de $8,5^{\circ} \mathrm{C}$, siendo la media de enero $15,2^{\circ} \mathrm{C}$ y la de julio $1,8^{\circ} \mathrm{C}$. Es común también que se presenten temperaturas inferiores a $0^{\circ} \mathrm{C}$ (Hajek \& Di Castri 1975). Los suelos corresponden a derivados de cenizas volcánicas y a suelos graníticos. Son de tipo impermeable-arcillosos, sometidos a una erosión permanente en el horizonte gumífero y con abundancia de clastos derrumbados debido a la alta pendiente (CONAF 2004).

Tanto desde el punto climático, como del ecológico, ésta es un área de transición, lo que le confiere una zonificación particular que se expresa en su vegetación (Peralta 1980). La vegetación representativa del área es un tipo de matorral bajo mediterráneo andino muy diverso, dominado por arbustos espinosos como Discaria chacaye (G. Don) Tortosa y Berberis empetrifolia Lam., los que frecuentemente se asocian a estratos herbáceos dominados por especies de los géneros Festuca, Stipa, Poa y otras especies acompañantes como Caltha sagittata Cav., Cerastium arvense L., Phacelia secunda J.F. Gmel., Senecio fistulosus Poepp. ex DC. y Sisyrinchium chilense Hook. (Luebert \& Pliscoff 2006).
Metodología

Durante los meses de enero de 2005 y marzo de 2006 se realizaron colectas intensivas en cinco veranadas al interior de la reserva: Cuchares Grande, Cajón Chileno, Tralilhue, Cuchares Chico y Mancha de Pinos. La descripción de cada sitio (ubicación, altitud y superficie) se muestra en la Tabla I. Estas cinco veranadas representan un gradiente descendente de pastoreo, correspondiendo a 218, 100, 54, 40 y 38 cabezas de ganado respectivamente, por cada temporada (promedio entre el periodo 2000-2008). En cada sitio de estudio se utilizó el método de transecto lineal. Cada transecto tenía un largo total de $20 \mathrm{~m}$, donde en cada $5 \mathrm{~m}$ se estableció una parcela al azar de 2 x 2 m (CONAMA \& TESAM 1996), en las que se determinó su composición de especies. Se realizaron 4 transectos aleatorios por cada veranada (20 parcelas en cada una), estableciendo un total de 100 parcelas en toda el área de estudio. La taxonomía, la nomenclatura y el origen fitogeográfico de las especies se obtuvo de Zuloaga et al. (2008) y Matthei (1995). Los nombres comunes se obtuvieron de Muñoz (1966) y Baeza (1930) y las formas de vida de Raunkiaer de las modificaciones propuestas por Ellenberg \& Mueller-Dombois (1966). El grado de intervención antrópico se determinó sobre la base de lo propuesto por Hauenstein et al. (1988), que consideran el origen fitogeográfico, es decir, la relación entre las especies nativas e introducidas y las formas de vida, como medidas de perturbación.

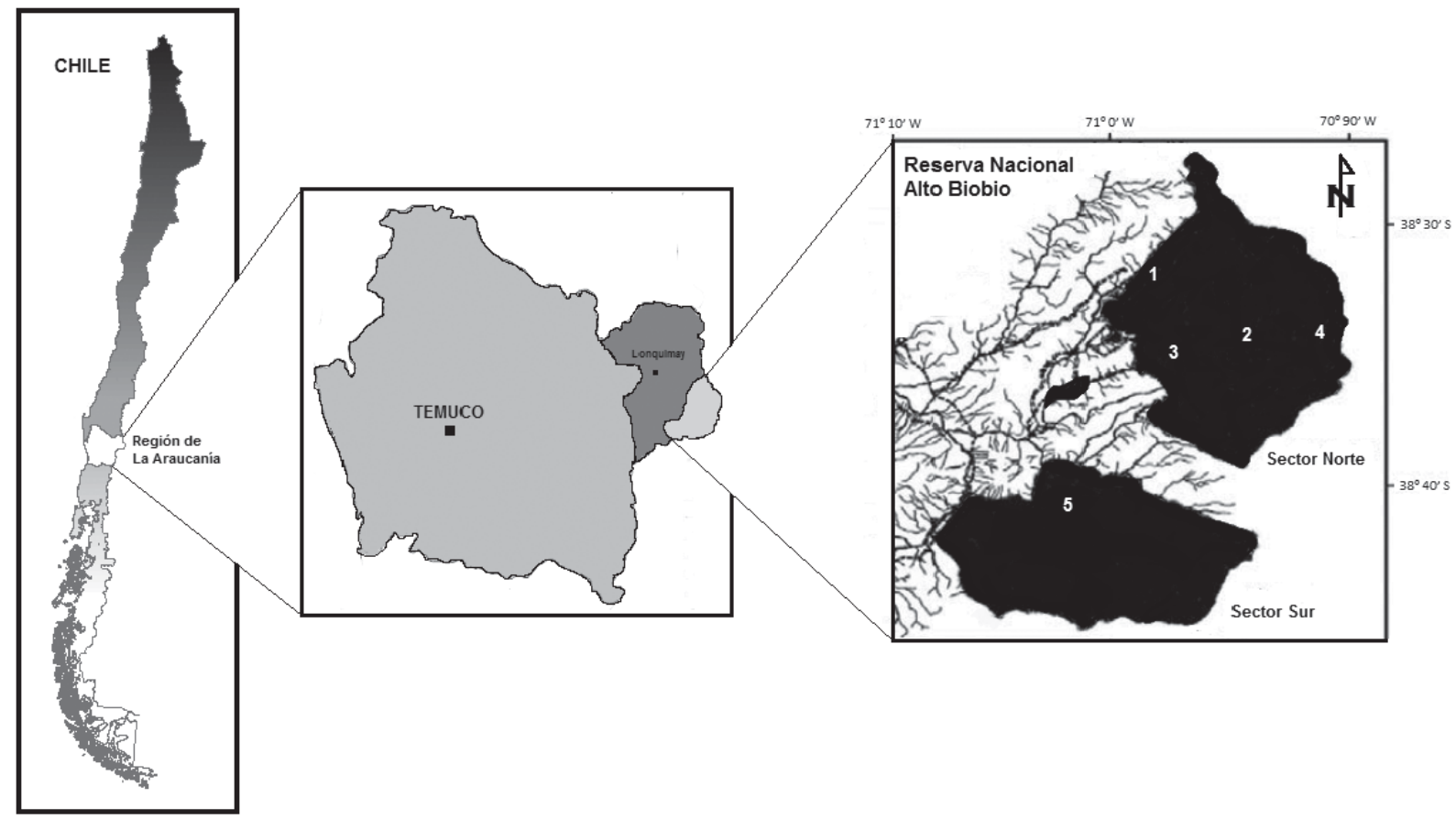

Figura 1. Área de estudio: Reserva Nacional Alto Bío-Bío, comuna de Lonquimay ( $\left.37^{\circ} \mathrm{S}\right)$, Chile. 1: Tralilhue, 2: Cuchares Grande, 3: Cuchares Chico, 4: Cajón Chileno, 5: Mancha de Pinos.

Figure 1. Study area: Alto Bío-Bío National Reserve, Lonquimay ( $\left.37^{\circ} \mathrm{S}\right)$, Chile. 1: Tralilhue, 2: Cuchares Grande, 3: Cuchares Chico, 4: Cajón Chileno, 5: Mancha de Pinos. 
Flora de veranadas en el Alto Bío-Bío: FuENTES-RAmírez, A. ET AL.

TABLA I. Ubicación y caracterización de los cinco sitios de muestreo al interior de la Reserva Nacional Alto Bío-Bío (37º S), Chile.

TABLE I. Location and characterization of the five study sites within the Alto Bío-Bío National Reserve ( $\left.37^{\circ} \mathrm{S}\right)$, Chile.

\begin{tabular}{|c|c|c|c|c|c|}
\hline SECTOR & VERANADA & Estaciones de Muestreo & $\begin{array}{c}\text { CoOrdenada GeOGRÁfica } \\
(\text { Lat S - Lon W) }\end{array}$ & $\begin{array}{l}\text { AltiTUd } \\
\text { (MSNM) }\end{array}$ & Superficie (HA) \\
\hline \multirow{8}{*}{ NORTE } & \multirow{2}{*}{ Tralilhue } & El Puesto & $38^{\circ} 32^{\prime} 2^{\prime \prime}-76^{\circ} 58^{\prime} 12^{\prime \prime}$ & 1.570 & \multirow{2}{*}{796} \\
\hline & & Vega de Tralilhue & $38^{\circ} 31^{\prime} 18^{\prime \prime}-76^{\circ} 57^{\prime} 40^{\prime \prime}$ & 1.610 & \\
\hline & \multirow{2}{*}{ Cuchares Grande } & El Puesto & $38^{\circ} 34^{\prime} 29^{\prime \prime}-76^{\circ} 54^{\prime} 6^{\prime \prime}$ & 1.480 & \multirow{2}{*}{1.425} \\
\hline & & Mallín Grande & $38^{\circ} 33^{\prime} 6^{\prime \prime}-76^{\circ} 54^{\prime} 17^{\prime \prime}$ & 1.560 & \\
\hline & \multirow{2}{*}{ Cuchares Chico } & El Puesto & $38^{\circ} 34^{\prime} 33^{\prime \prime}-76^{\circ} 55^{\prime} 5^{\prime \prime}$ & 1.735 & \multirow{2}{*}{348} \\
\hline & & Vega Cuchares Chico & $38^{\circ} 34^{\prime} 29^{\prime \prime}-76^{\circ} 56^{\prime} 18^{\prime \prime}$ & 1.630 & \\
\hline & \multirow{2}{*}{ Cajón Chileno } & El Puesto & $38^{\circ} 34^{\prime} 21^{\prime \prime}-76^{\circ} 50^{\prime} 38^{\prime \prime}$ & 1.805 & \multirow{2}{*}{564} \\
\hline & & Mallín Redondo & $38^{\circ} 33^{\prime} 49^{\prime \prime}-76^{\circ} 50^{\prime} 26^{\prime \prime}$ & 1.825 & \\
\hline \multirow{2}{*}{ SUR } & \multirow{2}{*}{ Mancha de Pinos } & Los Menucos & $38^{\circ} 43^{\prime} 57^{\prime \prime}-76^{\circ} 56^{\prime} 21^{\prime \prime}$ & 1.645 & \multirow{2}{*}{3.275} \\
\hline & & Los Menucos II & $38^{\circ} 44^{\prime} 5^{\prime \prime}-76^{\circ} 56^{\prime} 16^{\prime \prime}$ & 1.667 & \\
\hline
\end{tabular}

ANÁLISIS DE DATOS

Con los datos obtenidos se determinó la riqueza taxonómica, la riqueza total de especies, el origen fitogeográfico (OF) y las formas de vida (FV). Con esta clasificación se elaboró un catálogo de la flora con la descripción de cada especie. Adicionalmente, se realizó un análisis de similitud de BrayCurtis entre cada veranada utilizando Biodiversity-Pro 2.0 (McAleece 1997). Los ejemplares recolectados quedaron depositados en el Herbario de la Escuela de Ciencias Ambientales (UCT-Herbario no oficial), Facultad de Recursos Naturales, de la Universidad Católica de Temuco, Chile.

\section{RESULTADOS}

RiquEZA TAXONÓMICA

El estudio florístico reportó un total de 89 especies (Tabla III), distribuidas en 72 géneros y 37 familias. De las especies, dos corresponden a Pteridophyta (2\%) y 87 a Magnoliophyta (98\%). Éstas últimas se dividen en 25 Liliopsida (29\%) y 62 Magnoliopsida (71\%) (Tabla II, Fig. 2). La veranada de Cajón Chileno es la que presenta el mayor número de familias, géneros y especies. Por otro lado, Cuchares Chico es la que tiene el menor número de familias, géneros y especies (Tabla III). De las 37 familias registradas, sólo seis concentran más del 52\% de toda la flora registrada en el área de estudio: Asteraceae (13 spp.), Poaceae (11 spp.), Rosaceae (6 spp.), Cyperaceae (6 spp.) Juncaceae (5 spp.) y Apiaceae (5 spp.).
ORIGEN FITOGEOGRÁFICO Y FORMAS DE VIDA

Se registraron 78 especies nativas y 11 introducidas, representando un $88 \%$ y $12 \%$, respectivamente. La veranada de Cuchares Grande presentó 11 especies introducidas, Cajón Chileno nueve, Tralilhue ocho, Cuchares Chico 6 y Mancha de Pinos cinco (Fig. 3). Respecto de las formas de vida, 47 especies (52\%) corresponden a hemicriptófitos, seguidas de 15 criptófitos, 13 caméfitos, 8 nanofanerófitos y 6 terófitos. La proporción de especies nativas e introducidas para los caméfitos es 12 y 1, respectivamente, mientras que para los terófitos es 4 y 2, respectivamente. La veranada de Cuchares Grande es la que presenta la mayor proporción de especies introducidas (18,6\%) y la mayor proporción de hemicriptófitos con un 60\% (Fig. 3). Mancha de Pinos, por su parte, es la que presenta la mayor proporción de especies nativas $(90,4 \%)$ y menor proporción de hemicriptófitos con un $51 \%$ (Fig. 3).

\section{SimiLITUD}

La mayor similitud florística (basada en Bray-Curtis) se da entre la veranada de Tralilhue y Cuchares Grande con un 67\% y luego entre la veranada de Cajón Chileno y Cuchares Chico con un 64\% (Fig. 4). Por el contrario, las veranadas que comparten menos especies entre sí son Mancha de Pinos y Cuchares Grande con una similitud de 45\%.

Respecto del total de especies registradas en toda la reserva, la veranada de Mancha de Pinos es la que mejor representa, en términos de riqueza de especies y diversidad, la flora de las praderas andinas de la Reserva Nacional Alto Bío-Bío, ya que representa un 43\% de todas las especies registradas (Fig. 4). 
TABLA II. Riqueza taxonómica de las especies registradas en las veranadas de la Reserva Nacional Alto Bío-Bío (37 S), Chile.

TABLE II. Species richness by taxonomic groups in the andean grasslands of the Alto Bío-Bío National Reserve (37º S), Chile.

\begin{tabular}{lccc}
\hline Nivel TAXONÓMICO & FAMiLIAS & GÉNEROS & ESPECIES \\
\hline Pteridophyta & 2 & 2 & 2 \\
Magnoliophyta & 35 & 70 & 87 \\
$\quad$ Liliopsida & 6 & 20 & 25 \\
$\quad$ Magnoliopsida & 29 & 50 & 62 \\
\hline TOTAL & 37 & 72 & 89 \\
\hline
\end{tabular}

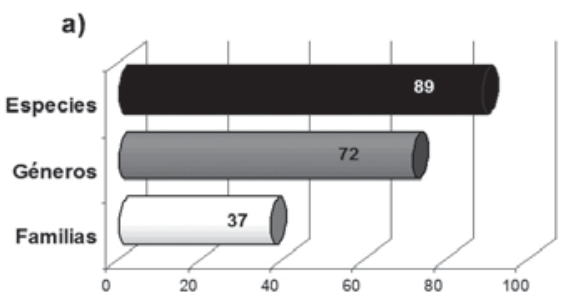

b)

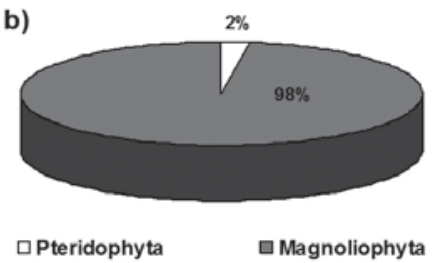

c)

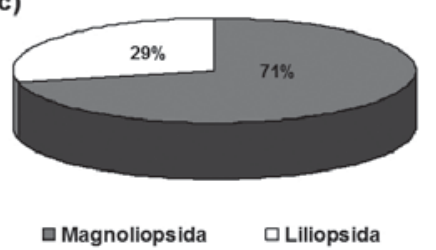

Figura 2. a) Número de especies, géneros y familias registradas en la Reserva Nacional Alto Bío-Bío (37º S), Chile. b) Distribución taxonómica general de las especies. c) Distribución de los taxa correspondientes a Magnoliophyta.

Figure 2. a) Numbers of species, genera and families registered in the Alto Bío-Bío National Reserve (37º S), Chile. b) General taxonomic distribution of the species. c) Distribution of Magnoliophyta taxa.

TABLA III. Distribución de la riqueza taxonómica de las especies registradas en cada sitio de estudio en las veranadas de la Reserva Nacional Alto Bío-Bío ( $\left.37^{\circ} \mathrm{S}\right)$, Chile. $\mathrm{F}$ = familias, G = géneros, E = especies.

TABLE III. Distribution of the taxonomic species richness in each study site in Alto Bío-Bío National Reserve ( $\left.37^{\circ} \mathrm{S}\right)$, Chile. F = families, $\mathrm{G}=$ genera, $\mathrm{E}$ = species.

\begin{tabular}{|c|c|c|c|c|c|c|c|c|c|c|c|c|c|c|c|}
\hline \multirow[b]{2}{*}{ Nivel TAXoNÓMico } & \multicolumn{3}{|c|}{ CuCHARES GRANDE } & \multicolumn{3}{|c|}{ CAJÓN ChILENo } & \multicolumn{3}{|c|}{ Tralilhue } & \multicolumn{3}{|c|}{ Cuchares Chico } & \multicolumn{3}{|c|}{ Mancha dE Pinos } \\
\hline & $\mathrm{F}$ & G & $\mathrm{E}$ & $\mathrm{F}$ & G & $\mathrm{E}$ & $\mathrm{F}$ & G & $\mathrm{E}$ & $\mathrm{F}$ & G & $\mathrm{E}$ & $\mathrm{F}$ & G & $\mathrm{E}$ \\
\hline Pteridophyta & - & - & - & 1 & 1 & 1 & - & - & - & 1 & 1 & 1 & - & - & - \\
\hline Liliopsida & 4 & 16 & 18 & 4 & 17 & 19 & 5 & 14 & 16 & 4 & 16 & 27 & 5 & 17 & 17 \\
\hline Magnoliopsida & 23 & 37 & 41 & 24 & 36 & 44 & 22 & 31 & 32 & 18 & 26 & 17 & 20 & 30 & 35 \\
\hline TOTAL & 27 & 53 & 59 & 29 & 54 & 64 & 27 & 45 & 48 & 23 & 43 & 45 & 25 & 47 & 52 \\
\hline
\end{tabular}


Flora de veranadas en el Alto Bío-Bío: FuENTES-RAmírez, A. ET AL.

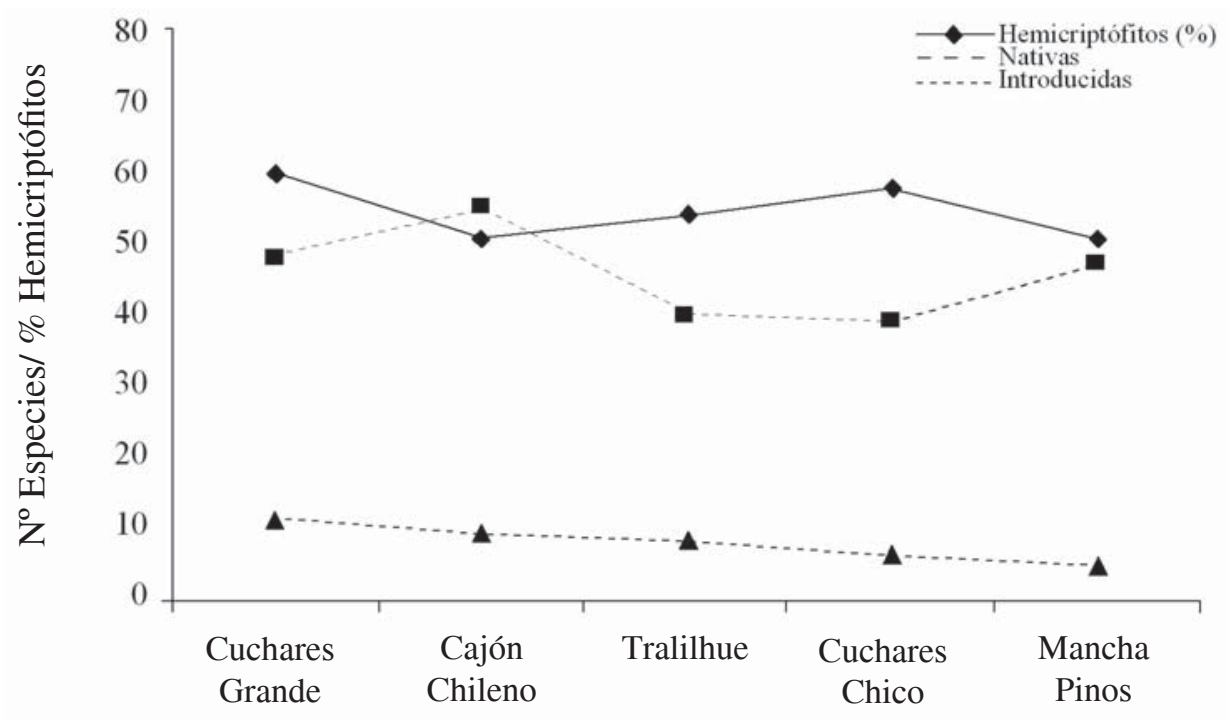

FIgURA 3. Número de especies nativas e introducidas y proporción (\%) de hemicriptófitos en cada sitio de estudio. Reserva Nacional Alto Bío-Bío (37º S), Chile.

Figure 3. Number of native and introduced species and the hemicryptophytes proportion (\%) in each study site. Alto Bío-Bío National Reserve ( $\left.37^{\circ} \mathrm{S}\right)$, Chile.
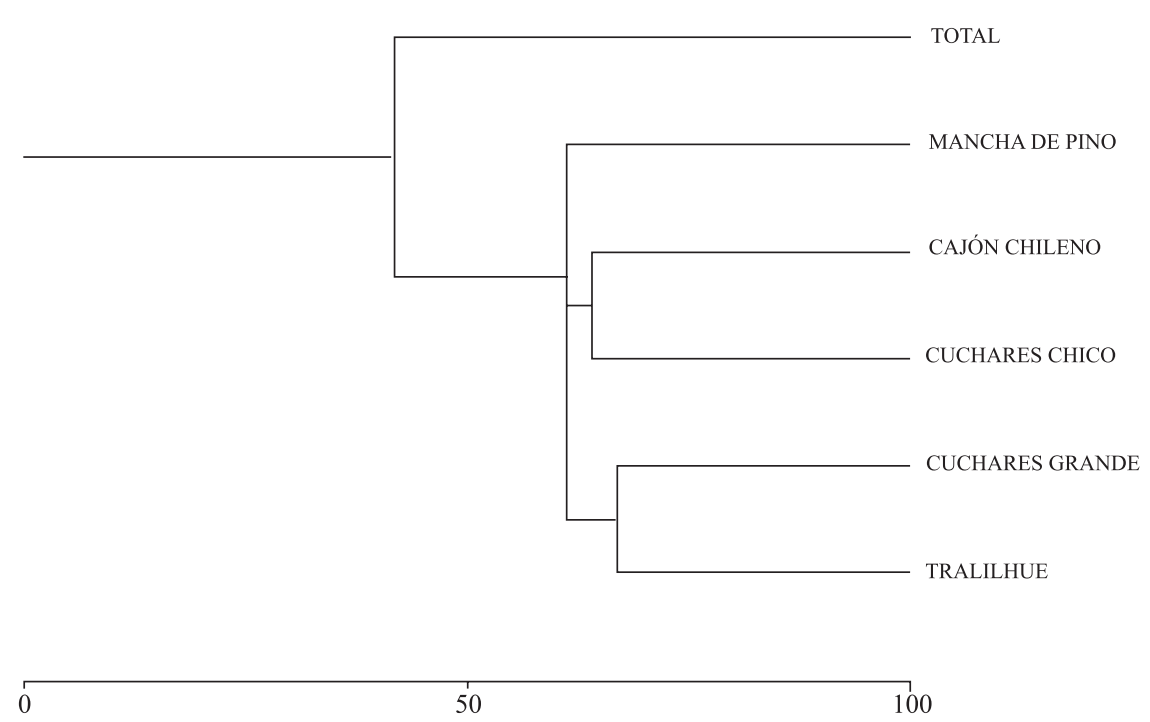

FiguRA 4. Dendrograma de similitud florística (Bray-Curtis) de las veranadas de la Reserva Nacional Alto Bío-Bío (37º S), Chile.

FIGURE 4. Floristic similarity dendrogram (Bray-Curtis) of the andean grasslands in the Alto Bío-Bío National Reserve (37º S), Chile. 
Gayana Bot. 68(1), 2011

TABLA IV. Catálogo de la flora vascular de las especies registradas en las veranadas de la Reserva Nacional Alto Bío-Bío (37º S), Chile. FV

= forma de vida, $\mathrm{OF}=$ origen fitogeográfico, $\mathrm{Hc}=$ hemicriptófito, $\mathrm{Ca}=$ caméfito, $\mathrm{Nf}=$ nanofanerófito, $\mathrm{Cr}=$ criptófito, $\mathrm{Te}=$ terófito, s.n. $=$ sin nombre.

TABLE IV. Vascular plants catalogue of the recorded species in the andean grasslands of the Alto Bío-Bío National Reserve (37 S), Chile. $\mathrm{FV}=$ life form, $\mathrm{OF}=$ fitogeographic origin, $\mathrm{Hc}=$ hemicryptophytes, $\mathrm{Ca}=$ chamaephytes, $\mathrm{Nf}=$ nanophanerophytes, $\mathrm{Cr}=$ cryptophytes, Te = terophytes, s.n.= without name.

\begin{tabular}{|c|c|c|c|c|}
\hline ESPECIE & FAMILIA & Nombre Común & $\mathrm{FV}$ & $\mathrm{OF}$ \\
\hline \multicolumn{5}{|l|}{ PTERIDOPHYTA } \\
\hline Ophioglossum crotalophoroides Walter & Ophioglossaceae & & $\mathrm{Cr}$ & $\mathrm{N}$ \\
\hline Polystichum andinum Phil. & Dryopteridaceae & & $\mathrm{Hc}$ & $\mathrm{N}$ \\
\hline \multicolumn{5}{|l|}{ MAGNOLIOPHYTA } \\
\hline \multicolumn{5}{|l|}{ A) Magnoliopsida } \\
\hline Acaena alpina Poepp. ex Walp. & Rosaceae & Trun & $\mathrm{Ca}$ & $\mathrm{N}$ \\
\hline Acaena macrocephala Poepp. & Rosaceae & & Hc & $\mathrm{N}$ \\
\hline Acaena magellanica (Lam.) Vahl. & Rosaceae & Amor seco, cadillo, trun & Hc & $\mathrm{N}$ \\
\hline Acaena ovalifolia Ruiz et Pav. & Rosaceae & Cadillo & Hc & $\mathrm{N}$ \\
\hline Acaena pinnatifida Ruiz et Pav. & Rosaceae & Cadillo, amor seco, pimpinela & Hc & $\mathrm{N}$ \\
\hline Adesmia emarginata Clos & Fabaceae & Paramela & $\mathrm{Ca}$ & $\mathrm{N}$ \\
\hline Anagalis alternifolia Cav. & Primulaceae & Pimpinela & Hc & $\mathrm{N}$ \\
\hline Anemone decapetala Ard. & Ranunculaceae & Centella & Hc & $\mathrm{N}$ \\
\hline Azorella monantha Clos & Apiaceae & Llareta & $\mathrm{Ca}$ & $\mathrm{N}$ \\
\hline Azorella trifurcata (Gaertn.) Pers. & Apiaceae & Llareta & $\mathrm{Ca}$ & $\mathrm{N}$ \\
\hline Baccharis magellanica (Lam.) Pers. & Asteraceae & & $\mathrm{Ca}$ & $\mathrm{N}$ \\
\hline Berberis empetrifolia Lam. & Berberidaceae & Zarcilla , monte negro & $\mathrm{Ca}$ & $\mathrm{N}$ \\
\hline Berberis montana Gay & Berberidaceae & Palo amarillo & $\mathrm{Nf}$ & $\mathrm{N}$ \\
\hline Calceolaria biflora Lam. & Calceolariaceae & Capachito & Hc & $\mathrm{N}$ \\
\hline Calceolaria dentata Ruiz et. Pav. & Calceolariaceae & Capachito & Hc & $\mathrm{N}$ \\
\hline Caltha sagittata Cav. & Ranunculaceae & Maillico & $\mathrm{Cr}$ & $\mathrm{N}$ \\
\hline Cerastium arvense $\mathrm{L}$. & Caryophyllaceae & Cuernecita & Hc & $\mathrm{I}$ \\
\hline Chiliotrichum diffusum (G.Forst.) Kuntze & Asteraceae & Romerillo, mata verde & $\mathrm{Nf}$ & $\mathrm{N}$ \\
\hline Cirsium vulgare (Savi) Ten. & Asteraceae & Cardo negro & $\mathrm{Te}$ & $\mathrm{I}$ \\
\hline Colletia hystrix Clos & Rhamnaceae & Crucero & $\mathrm{Nf}$ & $\mathrm{N}$ \\
\hline Dichondra sericea Sw. & Convolvulaceae & Oreja de ratón, pocha & Hc & $\mathrm{N}$ \\
\hline Discaria chacaye (G. Don) Tortosa & Rhamnaceae & Chacay & $\mathrm{Nf}$ & $\mathrm{N}$ \\
\hline Epilobium nivale Meyen & Onagraceae & Quilloy & $\mathrm{Hc}$ & $\mathrm{N}$ \\
\hline Erigeron andicola DC. & Asteraceae & & Hc & $\mathrm{N}$ \\
\hline Escallonia virgata (Ruiz et Pav.) Pers. & Escalloniaceae & Mata negra, meki & $\mathrm{Nf}$ & $\mathrm{N}$ \\
\hline Euphorbia collina Phil. & Euphorbiaceae & Pichoga & Hc & $\mathrm{N}$ \\
\hline Euphrasia andicola Benth. & Orobanchaceae & & $\mathrm{Ca}$ & $\mathrm{N}$ \\
\hline Euphrasia flavicans Phil. & Orobanchaceae & & Hc & $\mathrm{N}$ \\
\hline Galium aparine L. & Rubiaceae & Lengua de gato & Нc & $\mathrm{N}$ \\
\hline Gamocarpha alpina (Poepp.) H.V.Hansen & Calyceraceae & & Нc & $\mathrm{N}$ \\
\hline Gamochaeta purpurea (L.) Cabrera & Asteraceae & & $\mathrm{Te}$ & $\mathrm{N}$ \\
\hline Gaultheria pumila (L.f.) D.J.Middleton & Ericaceae & & $\mathrm{Ca}$ & $\mathrm{N}$ \\
\hline Gentianella magellanica (Gaudich.) Fabris ex D.M. Moore & Gentianaceae & & $\mathrm{Te}$ & $\mathrm{N}$ \\
\hline Gunnera magellanica Lam. & Gunneraceae & & $\mathrm{Cr}$ & $\mathrm{N}$ \\
\hline Hydrocotyle modesta Cham. et Schltdl. & Apiaceae & & Hc & $\mathrm{N}$ \\
\hline Hypochaeris radicata $\mathrm{L}$. & Asteraceae & Hierba del chancho & Hc & I \\
\hline Loasa sp. & Loasaceae & & $\mathrm{Te}$ & $\mathrm{N}$ \\
\hline
\end{tabular}




\begin{tabular}{|c|c|c|c|c|}
\hline Mimulus glabratus Kunth & Phrymaceae & Berro amarillo, placa & $\mathrm{Cr}$ & $\mathrm{N}$ \\
\hline Mulinum spinosum (Cav.) Pers. & Apiaceae & Neneo, dichillo & $\mathrm{Ca}$ & $\mathrm{N}$ \\
\hline Nassauvia aculeata (Less.) Poepp. et Endl. & Asteraceae & & $\mathrm{Ca}$ & $\mathrm{N}$ \\
\hline Nassauvia revoluta D. Don & Asteraceae & Cadislao & $\mathrm{Ca}$ & $\mathrm{N}$ \\
\hline Nothofagus antarctica (G.Forster.) Oerst. & Nothofagaceae & Ñirre, Ñire & $\mathrm{Nf}$ & $\mathrm{N}$ \\
\hline Oldenlanlia salzmanii (DC.) Benth. et Hook. & Rubiaceae & & Hc & $\mathrm{N}$ \\
\hline Phacelia secunda J.F.Gmel. & Boraginaceae & Cuncuna & Нс & $\mathrm{N}$ \\
\hline Plantago barbata G.Forster & Plantaginaceae & & Hc & $\mathrm{N}$ \\
\hline Plantago major L. & Plantaginaceae & Llantén & Нc & I \\
\hline Potentilla chiloensis (L.) Mabb. & Rosaceae & Frutilla & Hc & $\mathrm{N}$ \\
\hline Ranunculus peduncularis Sm. & Ranunculaceae & Botón de oro & Hс & $\mathrm{N}$ \\
\hline Ribes cucullatum Hook. et Arn. & Grossulariaceae & Parrilla & $\mathrm{Nf}$ & $\mathrm{N}$ \\
\hline Rumex acetosella L. & Polygonaceae & Vinagrillo & Hc & I \\
\hline Sanicula graveolens Poepp. ex DC. & Apiaceae & Cilantro del cerro, pata de león & Нc & $\mathrm{N}$ \\
\hline Senecio fistulosus Poepp. ex Less. & Asteraceae & Hualtata & Нc & $\mathrm{N}$ \\
\hline Senecio polygaloides Phil. & Asteraceae & & Нc & $\mathrm{N}$ \\
\hline Symphyotrichum glabrifolium (DC.) G.L.Nesom & Asteraceae & Margarita del pantano & Hc & $\mathrm{N}$ \\
\hline Taraxacum officinale G. Weber ex F.H. Wigg. & Asteraceae & Diente de león & Нc & I \\
\hline Trifolium pratense $\mathrm{L}$. & Fabaceae & Trébol rosado & $\mathrm{Ca}$ & I \\
\hline Trifolium repens L. & Fabaceae & Trébol blanco & Hс & I \\
\hline Vicia sp. & Fabaceae & & $\mathrm{Te}$ & $\mathrm{N}$ \\
\hline Viola reichei Skottsb. & Violaceae & Violeta & $\mathrm{Cr}$ & $\mathrm{N}$ \\
\hline \multicolumn{5}{|l|}{ B) Liliopsida } \\
\hline Agrostis capillaris L. & Poaceae & Chépica, pasto quila & Hc & I \\
\hline Alopecurus magellanicus Lam. & Poaceae & & Hc & $\mathrm{N}$ \\
\hline Carex fuscula D' Urv. var. fuscula & Cyperaceae & Cortadera & Нc & $\mathrm{N}$ \\
\hline Chloraea magellanica Hook.f. & Orchidaceae & & $\mathrm{Cr}$ & $\mathrm{N}$ \\
\hline Chusquea cf. culeou E. Desv. & Poaceae & Coligüe & $\mathrm{Nf}$ & $\mathrm{N}$ \\
\hline Cortaderia araucana Stapf & Poaceae & Cola de zorro & Нc & $\mathrm{N}$ \\
\hline Eleocharis radicans (Poir.) Kunth & Cyperaceae & & $\mathrm{Cr}$ & $\mathrm{N}$ \\
\hline Eleocharis macrostachya Britton & Cyperaceae & Rume & $\mathrm{Cr}$ & $\mathrm{N}$ \\
\hline Eleocharis melanostachys C.B. Clarke & Cyperaceae & & $\mathrm{Cr}$ & $\mathrm{N}$ \\
\hline Elymus angulatus J. Presl & Poaceae & & Hc & $\mathrm{N}$ \\
\hline Festuca scabriuscula Phil. & Poaceae & Coirón & Hc & $\mathrm{N}$ \\
\hline Holcus lanatus L. & Poaceae & Pasto miel, pasto dulce & $\mathrm{Te}$ & I \\
\hline Hordeum comosum J. Presl & Poaceae & Cebadilla & Нc & $\mathrm{N}$ \\
\hline Isolepis cernua (Vahl.) Roem. et Schult. & Cyperaceae & & $\mathrm{Cr}$ & $\mathrm{N}$ \\
\hline Juncus lesueurii Boland & Juncaceae & Junquillo & $\mathrm{Cr}$ & $\mathrm{N}$ \\
\hline Juncus pallescens Lam. & Juncaceae & Junquillo & Hc & $\mathrm{N}$ \\
\hline Juncus procerus E. Meyer & Juncaceae & Junquillo & Hc & $\mathrm{N}$ \\
\hline Luzula racemosa Desv. & Juncaceae & & $\mathrm{Cr}$ & $\mathrm{N}$ \\
\hline Marsippospermum philippii (Buch.) Haum. & Juncaceae & & $\mathrm{Cr}$ & $\mathrm{N}$ \\
\hline Oreobolus obtusangulus Gaudich. & Cyperaceae & & $\mathrm{Ca}$ & $\mathrm{N}$ \\
\hline Phleum alpinum L. & Poaceae & & Hс & I \\
\hline Poa tristigmatica E. Desv. & Poaceae & & Нc & $\mathrm{N}$ \\
\hline Rhodophiala andicola (Poepp.) Traub & Amaryllidaceae & Añañuca & $\mathrm{Cr}$ & $\mathrm{N}$ \\
\hline Rhodophiala montana (Phil.) Traub & Amaryllidaceae & Añañuca & $\mathrm{Cr}$ & $\mathrm{N}$ \\
\hline Sisyrinchium chilense Hook. & Iridaceae & Huilmo & Нc & $\mathrm{N}$ \\
\hline Trisetum spicatum (L.) K. Richt. & Poaceae & & Hc & $\mathrm{N}$ \\
\hline
\end{tabular}




\section{DISCUSIÓN}

RiQUEZA DE ESPECIES

En este estudio sólo se consideraron las praderas, vegas y mallines, dejando de lado la estepa, el bosque de Araucaria (Araucaria araucana) y Lenga (Nothofagus pumilio) y otras formaciones vegetales distintas a la pradera andina. En este sentido, el grupo de mayor riqueza, tanto en familias, géneros y especies es Magnoliophyta. De las 89 especies encontradas, el 98\% corresponden a Magnoliopsida y Liliopsida. Sólo dos especies corresponden a Pteridophyta, lo que se explica porque su hábitat preferente es el bosque (Godoy et al. 1982). Al comparar estos resultados con los de otros estudios similares, Dollens \& Ivanovic (1996) reportan un total de 52 especies para un sector de praderas del P.N. Torres del Paine. Su baja diversidad florística se explicaría por las condiciones climáticas más severas de la zona austral de Chile. En estudios realizados en veranadas de la misma zona del presente estudio, Saavedra et al. (2000) reportan 68 especies en la R.N. Nalcas y Hauenstein et al. (2003) registraron 117 especies en las veranadas de la zona de Lonquimay. Estos dos últimos estudios se acercan a los resultados obtenidos en la R.N. Alto Bío-Bío, ya que fueron realizados en formaciones vegetales y lugares geográficos similares. Es importante precisar que en el presente estudio se trabajó sólo en las praderas ubicadas al interior de esta Reserva, abarcando unas 3.000 ha aproximadamente. Asimismo, Hauenstein et al. (2003) reportan un patrón similar al encontrado en este estudio respecto de las familias más numerosas en términos de especies. En orden decreciente ellos reportan Poaceae (21), Asteraceae (16), Cyperaceae (7), Apiaceae (6), Rosaceae (5) y Juncaceae (4).

\section{ORIGEN FITOGEOGRÁFICO}

De las especies registradas en las veranadas de la R.N. Alto Bío-Bío, el 88\% son nativas y sólo el 12\% son introducidas. Esto sugiere que ellas presentarían una baja intervención antrópica debido a la escasa proporción de especies introducidas (González 2000). Sin embargo, este indicador debe ser complementado con la proporción entre las formas de vida tal como sugieren Hauenstein et al. (1988). Resultados similares en otras Áreas Silvestres Protegidas de Chile muestran que el Parque Nacional El Morado (3347' S; $70^{\circ} 04^{\prime} \mathrm{W}$ ), en la zona andina de Chile central, presenta un $13 \%$ de especies introducidas, mientras que el Parque Nacional Puyehue, en la Región de Los Lagos (40³0’S; $71^{\circ} 50^{\prime} \mathrm{W}$ ) presenta un 12\% de especies introducidas (Muñoz 1980, Pauchard \& Alaback 2004). Por el contrario, la flora del M. N. Cerro Ñielol, ubicado en Temuco en medio de una matriz urbana y agrícola, posee un 43\% de especies introducidas (Hauenstein et al. 1988). Estos datos confirman que la proporción de especies introducidas está fuertemente influenciada por el cambio de uso del suelo anterior a la creación del área protegida, las perturbaciones en la matriz circundante, el tráfico de visitantes y el desarrollo de la infraestructura de dichas unidades (Pauchard \& Alaback 2004). En zonas de veranadas, Saavedra et al. (2000) registran un 32\% de especies introducidas para la R. N. Nalcas. En la R. N. Malalcahuello, Becerra \& Faúndez (2001) reportan un 12\% y, finalmente, Hauenstein et al. (2003) en las veranadas de la zona de Lonquimay registraron un 20\%. En este estudio encontramos que aquellas veranadas con mayor carga animal (Cuchares Grande) son las que presentan mayor proporción de especies introducidas, en contraste con aquellas que tienen un uso menos intenso (Mancha de Pinos), que presentan mayor proporción de especies nativas. Existe un gradiente de especies introducidas que se correlaciona positivamente con el uso pastoril de cada veranada (Fig. 3). En la actualidad, las veranadas presentan serios problemas de sobrepastoreo (Pucheta et al. 1998, Florez 2005), lo que se evidencia por la alta frecuencia y cobertura encontrada para algunas especies introducidas como Trifolium repens L. (14,5\%) e Hypochaeris radicata L. (11\%) (Avendaño 2002). Estas dos especies introducidas son las que presentan mayor valor de importancia (Wikum \& Shanholtzer 1978) en las cinco praderas estudiadas: 32,3 y 19,5 respectivamente.

\section{FORMAS DE VIDA}

Hauenstein et al. (1988) plantean que para la determinación del grado de perturbación de un determinado lugar, el espectro biológico (formas de vida) sirve como complemento al origen fitogeográfico. De las 89 especies que crecen en las veranadas de la R.N. Alto Bío-Bío, 47 corresponden a hemicriptófitos. Estas especies están adaptadas a soportar el pisoteo y ramoneo de animales, representando así el 53\% del total de la flora registrada en las veranadas de la R.N. Alto Bío-Bío. Aunque en praderas de regiones templadas generalmente predominan estas formas de vida (Grigera et al. (1996), como lo señala Ramírez (1988) podrían también estar indicando algún grado de perturbación. Actualmente existe un uso ganadero creciente al interior de la reserva, lo que podría tener impactos sobre la composición florística de la pradera. Sin embargo, es posible que existan otros factores que den cuenta de la predominancia de esta forma de vida (principalmente el tipo de clima y la disponibilidad de recursos (Otto et al. 2001)). Cuchares Grande es la veranada con mayor presión de uso y la que presenta la mayor proporción de hemicriptófitos (60\%). Los criptófitos representados en un 17\%, incluyen a geófitos e hidrófitos, los que por poseer sus yemas de renuevo protegidas bajo tierra o en el agua, soportan mejor las bajas temperaturas del invernal. Los caméfitos están representados en un 15\%. Esta forma de vida es un indicador de condiciones de bajas temperaturas y precipitaciones en forma de nieve, comunes en la zona en época invernal (Huetz de Lemps 1983). La baja presencia de terófitos (7\%), plantas anuales o bianuales, indican ausencia de meses áridos, no obstante, en el área 
es posible que se registren 1 ó 2 meses secos (Hajek \& Di Castri 1975, Luebert \& Pliscoff 2006).

\section{SimiLITUD}

La veranada de Tralilhue y Cuchares Grande son las que presentan la mayor similitud en la composición de la flora (67\%), compartiendo 40 de 67 especies. Estas dos veranadas presentan una alta presión ganadera y son las más utilizadas en cada temporada (Fuentes-Ramírez com. pers.). Le siguen en similitud Cajón Chileno y Cuchares Chico (64\%), las que comparten 39 de 70 especies. Ambas veranadas también presentan una alta presión de uso, pero a la vez tienen un alto grado de aislamiento, comparado con las anteriores. Mancha de Pinos es la veranada que presenta la mayor similitud respecto del total de especies registradas en toda la reserva (41,6\%), por lo tanto es la que mejor representa la vegetación de las praderas andinas al interior de la reserva. Es la que se encuentra mejor conservada, la más aislada y la más extensa en superficie. También es la que presenta la presión de uso más baja, lo que se traduce en que tiene sólo un $12 \%$ de flora vascular introducida. De este último análisis se desprenden las menores similitudes florísticas, que precisamente se dan entre la veranada de Mancha de Pinos y Cuchares Grande (45\%). El contraste entre floras podría responder a las diferencias en la carga animal que soportan estas veranadas cada temporada. Entre los efectos más comunes que produce el pastoreo sobre la estructura de pastizales naturales están los cambios en la estructura, diversidad florística y formas de vida, la disminución del establecimiento y del crecimiento de la vegetación. (Milchunas \& Lauenroth 1993, Pettit et al. 1995, Pucheta et al. 1998, Stohlgren et al. 1999a, Nai-Bregaglio et al. 2002).

Los trabajos que evalúan el impacto de la ganadería en praderas andinas o veranadas son escasos. Sólo existen algunos antecedentes generales en la Región de Coquimbo, los cuales señalan que el sobre pastoreo, en particular el caprino, produce empobrecimiento de la pradera, erosión del suelo y llegada de especies introducidas potencialmente invasoras (Olivares 2006). A pesar de no existir registros de pastoreo caprino en las veranadas de la R. N. Alto Bío-Bío, sí es posible encontrar algunos rebaños pastando en zonas aledañas (Fuentes-Ramírez com. pers.), representando así un riesgo para la conservación de dichos recursos. Por otro lado, Ramírez et al. (1997, 2005), en praderas antropogénicas del sur de Chile, establecen que el pastoreo incrementa la riqueza de especies, pero a la vez acarrea especies introducidas que a largo plazo pueden invadir la pradera. En nuestro estudio, el patrón encontrado es similar ya que en praderas con mayor carga animal la riqueza de especies es mayor, pero algunas especies introducidas presentan alta frecuencia y cobertura (Trifolium repens e Hypochaeris radicata). La presión de pastoreo sobre las veranadas de la Reserva Nacional Alto Bío-Bío ha aumentado sistemáticamente en los últimos años. Por ende, se hace necesario un manejo adecuado a fin de asegurar su permanencia y calidad para beneficio de las comunidades pehuenches aledañas.

\section{CONCLUSIONES}

A pesar de presentar una baja proporción de especies vegetales introducidas, las veranadas de la Reserva Nacional Alto Bío-Bío exhiben un grado medio de perturbación debido al aumento del pastoreo, que a su vez incrementa la presencia de especies hemicriptófitas. Existe, además, una alta similitud entre praderas de uso ganadero intensivo como Tralilhue y Cuchares Grande y una baja similitud con aquéllas de menor carga animal (Mancha de Pinos). Se concluye que el pastoreo sería una de las principales causas que estarían dando cuenta del cambio florístico observado entre las praderas estudiadas. Sin embargo, a través del manejo adecuado de praderas se podrían evitar impactos mayores frente a futuros escenarios de cambio global.

\section{AGRADECIMIENTOS}

Agradecemos a Eliecer Ñancufil y Heraldo Meliqueo, funcionarios de CONAF Región de La Araucanía y encargados de la Reserva Nacional Alto Bío-Bío por el apoyo brindado en terreno. A Basilio Guíñez, Pamela Sánchez y Marcos González de la Universidad Católica de Temuco. A Pedro Jara-Seguel y dos revisores anónimos por sus contribuciones en el manuscrito. AFR y AP fueron financiados por PFB-23 e ICM P05-002.

\section{BIBLIOGRAFÍA}

Adler, P.B., D.G. Milchunas, O.S. Sala, I.C. Burke \& W.K. LAUENROTH. 2005. Plant traits and ecosystem grazing effects: comparison of U.S sagebrush steppe and Patagonian steppe. Ecological Applications 15: 774-972.

Ahumada, M., R. Palma, A. Centrón, S, Ramírez, E. Hauenstein, M. GonzÁlez \& G. Pérez. 1999. Pauta de condición de las veranadas en la IX Región de La Araucanía. Ministerio de Agricultura (SAG). Departamento de Protección de los Recursos Naturales Renovables 9: 1-71.

Avendaño, M. 2002. Evaluación preliminar de la capacidad de carga de los pastizales altoandinos en una zona de la Reserva Nacional Salinas y Agua Blanca. Libro resúmenes $3^{\text {er }}$ Congreso Latinoamericano de Manejo de Cuencas Hidrográficas. 8-13 junio, 2003. Arequipa. Perú. 430 pp.

BAEzA, V.M. 1930. Los nombres vulgares de las plantas silvestres de Chile y su concordancia con los nombres científicos. Y observaciones sobre la aplicación técnica y medicinal de algunas especies. $2^{\mathrm{a}}$ ed. Santiago. $270 \mathrm{pp}$.

Becerra, P. \& L. Faúndez. 2001. Diversidad florística de la Reserva Nacional Malalcahuello, IX Región, Chile. 
Revista electrónica Chloris chilensis. Año 2, Nº1. URL: http://www.chlorischile.cl. Texto en formato PDF. 10 pp.

Bustamante, P. 2007. Veranadas: situación actual y temas pendientes. Programa Ambiental DAS CuracautínLonquimay. Obispado de Temuco. Chile. 24 pp.

Castro, S.A., E. Badano, D. Guzmán \& L.A. Cavieres. 2010. Biological invasion of a refuge habitat: Anthriscus caucalis (Apiaceae) decreases diversity, evenness, and survival of native herbs in the Chilean matorral. Biological Invasions 12: $1295-1303$.

Comisión Nacional del Medio Ambiente \& Tesam. 1996. Metodologías para la caracterización de la Calidad Ambiental. Chile. 239 pp.

Corporación NaCional Forestal. 2000. Reglamento de uso de las praderas andinas en las Reservas Nacionales de la IX Región. Ministerio de Agricultura. Unidad de Gestión de Patrimonio Silvestre. Unidad de Operaciones. CONAF. Chile. 4 pp.

Corporación NACIONAL Forestal. 2004. Antecedentes para el plan de manejo de la Reserva Nacional Alto Bío-Bío. Unidad de Gestión de Patrimonio Silvestre. Sección Parques y Monumentos. CONAF. Chile. 19 pp.

Davis, M.A., J.P. Grime \& K. Thompson. 2000. Fluctuating resources in plant communities: a general theory of invasibility. Journal of Ecology 88: 528-534.

Dollens, O. \& J. Ivanovic. 1996. Sucesión secundaria en un pastizal incendiado en el Parque Nacional Torres del Paine. Anales Instituto de la Patagonia. Serie Ciencias Naturales 24:15-28

Ellenberg, H. \& D. Mueller-Dombois. 1966. A key to Raunkiaer plant life forms with revised subdivisions. Berichte Geobotanisches Institut ETH Stiftung Rubel. Zurich. 37: 56-73.

Florez, A. 2005. Manual de pastos y forrajes altoandinos. ITDG AL. OIKOS. Lima. Perú. 53 pp.

Godoy, R., C. Ramírez, H. Figueroa \& E. Hauenstein. 1982. Estudios ecosociológicos en Pteridófitos de comunidades boscosas valdivianas, Chile. Bosque 4: 12-24.

GonzÁlez, A. 2000. Evaluación del recurso vegetacional en la cuenca del río Budi, situación actual y propuestas de manejo. Tesis Licenciatura en Recursos Naturales. Facultad de Ciencias. Universidad Católica de Temuco, Chile. 110 pp.

Grigera, D., C. Brion, J.O. Chiapella \& M.S. Pillado. 1996. Las formas de vida de las plantas como indicadores de factores ambientales. Medio Ambiente 13: 11-29.

Hajek, E. \& F. Di Castri. 1975. Bioclimatografía de Chile. Dirección de Investigación. Pontificia Universidad Católica de Chile. Chile. 225 pp.

Hauenstein, E., C. Ramírez, M. Latsague \& D. Contreras. 1988. Origen fitogeográfico y espectro biológico como medida del grado de intervención antrópica en comunidades vegetales. Medio Ambiente 9: 140-142.

Hauenstein, E., R. Palma, M. González \& M. Ahumada. 2003. Composición florística de praderas altoandinas de la zona de Lonquimay (IX Región, Chile). Agro Sur 31: 8-20.

Hoffmann, A. 1978. Flora Silvestre de Chile. Zona Central. Ediciones Fundación Claudio Gay. Santiago. Chile. 255 pp.

Hoffmann, A. 1982. Flora Silvestre de Chile. Zona Araucana.
Ediciones Fundación Claudio Gay. Santiago. Chile. 257 pp.

Hoffmann, A., M.T.K. Arroyo, F. Liberona, M. Muñoz \& J. WATson. 1998. Plantas altoandinas en la Flora Silvestre de Chile. Ediciones Fundación Claudio Gay. Santiago. Chile. $281 \mathrm{pp}$.

Huetz DE Lemps, A. 1983. La vegetación de la Tierra. Editorial Akal-Universitaria. Barcelona. España. 263 pp.

Instituto Geográfico Militar. 1986. Geografía de la IX Región. Ediciones del IGM. Santiago. Chile. 250 pp.

Koeppen, W. 1948. Climatología. Fondo de Cultura Económica. México. 478 pp.

LarCher, W., C. Kainmüller \& J. Wagner. 2010. Survival types of high mountains plants under extreme temperatures. Flora. 205: 13-18.

LÓPEz, R. 1990. Estudio preliminar sobre ordenación de veranadas y la exclusión de ganado doméstico. III etapa. Regiones VII, VIII, IX. Ministerio de Agricultura. Servicio Agrícola y Ganadero. DIPROREN. 116 pp.

Luebert, F. \& P. Pliscoff. 2006. Sinopsis bioclimática y vegetacional de Chile. Editorial Universitaria. Santiago. Chile. 316 pp.

Mardones, M., M. Rondanelli, E. Ugarte \& A. Rodríguez. 1993. Planificación ecológica en el sector Icalma-Liucura (IX Región): Proposición de un método. Centro EULA. Universidad de Concepción. Chile. Serie Monografías Científicas. Vol. 6. 92 pp.

Matthei, O. 1995. Manual de las malezas que crecen en Chile. Alfabeta Impresores. Santiago, Chile. 545 pp.

McAleece, N. 1997. Biodiversity Professional Beta 1. Version 2.0. The Natural History Museum and the Scottish Association for Marine Science.

Milchunas, D. \& W. Lauenroth. 1993. A quantitative assessment of the effects of grazing on vegetation and soils over a global range of environments. Ecological Monographs 63: 327-366.

Molina, R. \& M. Correa. 1998. Territorio y comunidades Pehuenches del Alto Bío-Bío. Corporación Nacional de Desarrollo Indígena. Chile. 225 pp.

MuÑoz, M. 1980. Flora del Parque Nacional Puyehue. Editorial Universitaria. Santiago, Chile. 557 pp.

MuÑoz, C. 1966. Sinopsis de la flora chilena. Ediciones Universidad de Chile, Santiago. 500 pp.

Nai-Bregaglio, M., E. Pucheta \& M. Cabido. 2002. El efecto del pastoreo sobre la diversidad florística y estructural en pastizales de montaña del centro de Argentina. Revista Chilena de Historia Natural 75: 613-623.

Olivares, A. 2006. Pastizales y producción animal en zonas áridas de Chile. Secheresse (Montrougue) 17: 257-264.

Otto, R., J.M. Fernández-Palacios, B.O. Krusi. 2001. Variation in species composition and vegetation structure of succulent scrub on Tenerife in relation to environmental variation. Journal of Vegetation Science 12: 237-248.

Pauchard, A. \& P.B. Alaback. 2004. Influence of elevation, land use, and landscape context on patterns of alien plant invasions along roadsides in protected areas of southcentral Chile. Conservation Biology 18: 238-248.

Pauchard, A., L.A. Cavieres, R. Bustamante, P. Becerra \& E. RAPOPORT. 2004. Increasing the understanding of plant invasions in southern South America: first symposium on 
Alien Plant Invasions in Chile. Biological Invasions 6: 255-257.

Peralta, M. 1980. Geomorfología, clima y suelos del tipo forestal Araucaria en Lonquimay. Facultad de Ciencias Forestales, Universidad de Chile. Boletín Técnico № 57. Santiago, Chile. 15 pp.

Pérez, R. 2001. Descripción de la veranada de Pulul. Informe de Práctica para optar al título de Ingeniero de Ejecución Agrícola. Universidad de la Frontera. Temuco, Chile. 54 pp.

Pettit, N., R. Froend \& P. Ladd. 1995. Grazing in remnant woodland vegetation: changes in species composition and life form groups. Journal of Vegetation Science 6: 121130.

Pucheta, E., M. Cabido, S. Díaz \& G. Funes. 1998. Floristic composition, biomass, and aboveground net plant production in grazed and protected sites in a mountain grassland of central Argentina. Acta Oecologica 19: 97105.

PyкÄLÄ, J. 2004. Cattle grazing increases plant species richness of most species trait groups in mesic semi-natural grasslands. Plant Ecology 175: 217-226.

Ramírez, C. 1988. Formas de vida, fitoclimas y formaciones vegetales. El Árbol Nuestro Amigo 4: 33-37.

Ramírez, C., C. San Martín, A. Ellies \& R. Mac Donald. 1997. Cambios florísticos, fitosociológicos y edáficos provocados por exclusión de pastoreo en una pradera valdiviana, Chile. Agro Sur 25: 180-195.

Ramírez, C., C. San Martín \& C. Grüner. 2005. Cambios florísticos $\mathrm{y}$ vegetacionales con diferentes manejos pecuarios en un suelo andeptic palehumults (La Unión, X Región, Chile). Agro Sur 33: 13-28.

Saavedra, M., E. Hauenstein, C. Ramírez, C. San Martín, M. GonzÁlez, J. Burgos, J. Amigo \& S. Meza. 2000. Estudio florístico en veranadas afectadas por talajeo de la Reserva
Nacional Nalcas y recomendaciones para su conservación. Ministerio de Agricultura. CONAF IX Región. Boletín Técnico $\mathrm{N}^{\circ}$ 70. Temuco, Chile. 19 pp.

Servicio Agrícola y Ganadero. 2004. Las praderas en Chile y su relación con el desarrollo ganadero. Boletín Departamento de protección de los recursos naturales renovables. Vol. I, $\mathrm{N}^{\mathrm{o}} 2.5 \mathrm{pp}$.

Stohlgren, T.J., L.D. Schell \& B. V. Heuvel. 1999a. How grazing and soil quality affect native and exotic plant diversity in rocky mountain grasslands. Ecological Applications 9:4564.

Tacón, A., U. Fernández \& F. Ortega. 2002. El mercado de los productos no maderables y su papel en la conservación de la Ecorregión de los Bosques Valdivianos. Chile. 144 pp.

Ugarte, E. \& C. Barrientos. 1991. La vegetación de la cuenca del Alto Bío-Bío I: Una aproximación fisonómico- estructural. Revista Geográfica de Chile. Terra Australis 35: 67-79.

Ugarte, E., R. Boerner \& C. Barrientos. 1993. La vegetación de la cuenca del Alto Bío-Bío II: Comunidades, clasificación y dinámica. Gayana Botánica 50: 103-110.

VentuRA, O. 2001. Valoración económica de los bienes y servicios ambientales de las praderas altoandinas en el Perú: Políticas para el manejo sostenible. Libro resúmenes $3^{\text {er }}$ Congreso Latinoamericano de Manejo de Cuencas Hidrográficas. 813 junio, 2003. Arequipa. Perú. 430 pp.

Wikum, D. \& G. Shanholtzer. 1978. Aplication of the BraunBlanquet cover-abundance scale for vegetation analysis in land development studies. Environmental Management 2: 323-329.

Zuloaga, F.O., O. Morrone \& M. Belgrano. 2008. Catálogo de las Plantas Vasculares del Cono Sur. Monographs in Systematic Botany 107, Missouri Botanical Garden Press, St. Louis, Missouri, USA. 3384 pp.

Recibido: 08.06.10

Aceptado: 09.12.10 